\title{
Movimientos de mujeres indígenas en América Latina: el caso de Panamá y la CONAMUIP
}

\author{
Ma Dolores GonzÁlez Guardiola \\ Universidad de Castilla-La Mancha \\ Dolores.Gonzalez@uclm.es
}

Recibido: 2 de diciembre de 2010

Aceptado: 28 de enero de 2011

\begin{abstract}
RESUMEN
La Coordinadora Nacional de Mujeres Indígenas de Panamá es la máxima representante de las mujeres indígenas de Panamá (pueblos kuna, emberá, wounaan, ngobe, buglé, naso y bri-bri). Su creación, evolución y desarrollo ha de ser contemplado en el contexto del protagonismo creciente de los pueblos indígenas de América Latina, así como en el proceso de fortalecimiento del Movimiento de Mujeres de Panamá desde el inicio de la década de los 90 . Su construcción se ha realizado paralelamente a través de itinerarios comunitarios específicos (Coordinadoras Comarcales), instancias nacionales -Consejo Nacional de la Mujer(CONAMU) y la Dirección Nacional de la Mujer(DINAMU) de Panamá-y espacios internacionales (Enlace Continental de Mujeres Indígenas de las Américas, ECMIA) constituyéndose como una organización interétnica cuyo objetivo es el trabajo con y desde las mujeres indígenas en todos los lugares en los que estas se encuentren (comunidades, comarcas, barriadas). Las tareas y proyectos realizados, encaminados al fortalecimiento individual y colectivo, han abarcado todos los temas que afectan a los pueblos indígenas y, especialmente, a las mujeres indígenas (interculturalidad, violencia, salud, conocimientos tradicionales de los pueblos indígenas, medio ambiente, etc.).
\end{abstract}

Palabras clave: Movimientos de mujeres indígenas, CONAMUIP, Panamá, estudios de Género.

\section{Indigenous Women's Movements in Latin America: the Case of Panama and CONAMUIP}

\begin{abstract}
The National Coordination of Indigenous Women of Panama (CONAMUIP) is the most important representative of the indigenous women of Panama (peoples of Kuna, Emberá, Wounaan, Ngobe, Buglé, Naso and Bri-Bri). The creation, evolution and development of the CONAMUIP must be considered in the context of the increasing prominence of the Indigenous Peoples in Latin America, as well as in the process of strengthening of the Women's Movement of Panama since the beginning of the 90's. The construction of the National Coordination has been simultaneously carried out through specific community itineraries (Regional Coordinators), national authorities -National Council for Woman (CONAMU) and National Directorate of Woman (DINAMU)- and international networks (Continental Network of Indigenous Women of the Americas, ECMIA). Thus, the CONAMUIP has set itself up in an inter-ethnic organization whose goal is working with and from indigenous women within all the places where they live (communities, counties, suburban areas). The tasks and projects, aimed for the individual and collective empowerment, have covered all kind of issues that affect indigenous peoples and especially indigenous women (such as interculturalism, violence, health, traditional knowledge of indigenous peoples, environment, etc.).
\end{abstract}

Keywords: Indigenous Women's Movements, CONAMUIP, Panama, Gender Studies.

Sumario: 1. La mirada antropológica y los discursos de la igualdad. 2. Movimientos de Mujeres Indígenas. 3. Panamá y la CONAMUIP. 4. Conclusiones. 5. Referencias bibliográficas. 
El enfoque específico de la antropología nos ubica en el espacio de la diferencia, de la «otredad»y, por tanto, en los estudios de diversidad cultural. Sus planteamientos nos permiten reconocer las posturas adoptadas por otros sujetos sociales que visibilizan e incorporan sus propios enfoques a las estrategias, demandas y luchas por la igualdad y la equidad. De la misma manera, la producción teórica de feministas latinoamericanas, africanas y asiáticas reclama un reconocimiento de la pluralidad y la diversidad a partir del cuestionamiento del colonialismo discursivo del feminismo occidental, al mismo tiempo que surgen nuevas propuestas conceptuales para el reconocimiento de todas las formas de la desigualdad. Desde instancias múltiples se han levantado voces con propuestas autónomas que reclaman participar en el debate, estableciendo un diálogo a partir de sus propios principios culturales y sus propias condiciones socioeconómicas en contextos históricos específicos. Entre esas voces se encuentra, como un ejemplo destacado, la Coordinadora Nacional de Mujeres Indígenas de Panamá (CONAMUIP). Este artículo es fruto de una trayectoria orientada a la investigación y especialización en el estudio de los Movimientos de Mujeres en América Latina y especialmente a los Movimientos de Mujeres Indígenas ${ }^{1}$. Las estancias más recientes que he realizado en Guatemala y Panamá son la base para la profundización en el conocimiento de sus últimas actuaciones y tendencias en el contexto de la emergencia de los pueblos indígenas de América Latina $^{2}$. Todo ello conforma un proceso que se concreta en momentos y lugares determinados y en el que se inscriben las relaciones establecidas con la Coordinadora Nacional de Mujeres Indígenas de Panamá (CONAMUIP). Su anuencia y aceptación me permitieron compartir su trabajo en el verano de 2010. En este período tuve la ocasión de poder comprender y aprender, en cierta medida, el transcurrir diario de la propia organización, compartir los eventos en los que tomaron parte y escuchar sus problemas, demandas y luchas. Necesariamente tengo que comenzar, por tanto, agradeciendo su recibimiento y acogida a todas las mujeres ${ }^{3}$ de la Coordinadora con las que tuve ocasión de compartir un tiempo intenso y, especialmente, quiero mostrar mi agradecimiento y mi reconocimiento a Sonia Henríquez a la que se puede considerar auténtica memoria histórica del movimiento de mujeres indígenas de Panamá y que en la actualidad es la Presidenta General de la CONAMUIP. En este sentido quiero decir que este trabajo se plantea como un espacio de visibilización de

\footnotetext{
1 Una trayectoria ligada desde sus comienzos a los movimientos generados por mujeres de la ciudad de El Alto en respuesta a los mecanismos de la donación alimentaria en Bolivia.

2 Dichas estancias se han desarrollado en el marco del Programa de Ayudas en Cooperación para el Desarrollo, convocado anualmente por la Universidad de Castilla-La Mancha, y me han permitido sentar las bases para desarrollar dos proyectos de investigación: el primero, verificado en Guatemala durante un mes en el verano de 2009, abordó el tema de la alfabetización de las mujeres indígenas y la participación comunitaria femenina; el segundo, también llevado a cabo durante un mes en el verano de 2010, se basó en el apoyo a las actividades formativas de la Coordinadora Nacional de Mujeres Indígenas de Panamá (CONAMUIP).

3 Este trabajo se basa en las entrevistas realizadas a Sonia Henríquez (Presidenta de CONAMUIP) y a Ebinia Santos (Representante de la Coordinadora General de Mujeres Ngobe) así como a las conversaciones mantenidas con algunas de las integrantes de la Coordinadora. Mi especial agradecimiento a Sonia Henríquez y a Ebinia Santos, así como a Zuleika Arosamena, Eladia Carpintero, Fanny Méndez, Graciela Arias, Elvira Guillén y al resto de mujeres que tuve ocasión de conocer. También mi agradecimiento a Naguibler Henríquez por su apoyo y ayuda.
} 
la propia Coordinadora y también como un ejercicio consensuado de construcción colectiva del conocimiento basado en la capacidad de interlocución generada en este proceso.

\section{La mirada antropológica y los discursos de la igualdad}

En este espacio y en este tiempo que es la España de las últimas décadas, la diversidad cultural se ha colado en nuestras casas, en nuestras retinas, en nuestras conversaciones, en nuestras rutinas diarias, e incluso hasta en nuestras preocupaciones y nos ha generado expectativas, curiosidad, aunque también difusas o claras sensaciones de incomodidad. Por otro lado, pocas exposiciones, conferencias, cursos, enfoques y perspectivas de análisis de cada realidad sociocultural, en ámbitos académicos e intelectuales, ignoran las nociones de multiculturalidad, pluriculturalidad e interculturalidad y la hacen explícita de una u otra manera. Al fin y al cabo es una realidad que se ha instalado con fuerza, con rotundidad, con el ímpetu de los que deciden, de los que necesitan buscar más allá de su propia realidad y nos muestran directamente otras caras del mundo. En este contexto las nociones, que han sido herramientas conceptuales centrales en la antropología, se han expandido, han permeado y traspasado las aulas y los despachos y se utilizan con relativa normalidad en el lenguaje cotidiano. El concepto generalizado de cultura se amplía incluyendo la concepción característica de la antropología y se habla de otras culturas o de nuestras visiones etnocéntricas. Y todo ello multiplicado y aumentado en un mundo donde todo se dice, donde se dice a través de mil distintos canales de comunicación y donde se transmite, se difunde, se informa y se comunica a una enorme cantidad de gente muy diversa. Algo impensable hace bien poco tiempo. En definitiva se visibiliza aquello que no hace mucho tiempo conocíamos de oídas, a través de viajeros de todo tipo y condición (comerciantes, soldados, aventureros) que relataban costumbres extrañas, exóticas e increíbles.

Además, la descripción del contexto que envuelve el objetivo de la reflexión que aquí se realiza exige identificar también (a modo de círculos concéntricos) el desarrollo de los denominados discursos de la igualdad de género, entendiendo por tales al conjunto de argumentaciones y herramientas conceptuales, teóricas y metodológicas que se han elaborado demandando la igualdad/equidad entre hombres y mujeres y que fue históricamente encabezada por feministas occidentales. Utilizo esta denominación, por tanto, como marco contextual y como forma de resumir el conjunto de dichos planteamientos que también paulatinamente de manera cada vez más global, se han extendido a lo largo de la segunda mitad del siglo XX. Su importancia, al menos teórica, se demuestra en su presencia en los más diferentes espacios: movimientos por la igualdad; desarrollos legislativos; políticas locales, nacionales y supranacionales diversas; estrategias de desarrollo y líneas de cooperación; presencia en espacios académicos, etc. Estos discursos distan de ser homogéneos, entre otras cosas por su carácter cada vez más complejo, y por tanto deben ser identificados porque conviene reconocer a los/las generadores/as de los mismos como una vía imprescindible para 
establecer correspondencias entre los diferentes actores sociales que como depositarios o usuarios se nutren de ellos y para evaluar correctamente su impacto. En este armazón de gran complejidad, en esta urdimbre de múltiple trama, que en muchas ocasiones no es más que una yuxtaposición de iniciativas a modo de tejido de patchwork, hemos podido cada vez distinguir con más fuerzas otros planteamientos, otras iniciativas.

La importancia adquirida por este conjunto de argumentaciones, así como los efectos causados y los impactos producidos, ha contribuido a que sea considerado un tema central que es objeto de atención por parte de los analistas y que debe seguir siendo tratado, estudiado y debatido. Debe ser así porque, entre otras cosas, parece haber un cierto acuerdo en que después de décadas de elaboración y de esfuerzo para que sus planteamientos fueran escuchados, sigue existiendo una importante brecha entre la igualdad formal y la igualdad real. Por otro lado, la riqueza innegable de sus planteamientos, cuyo eje central ha sido la incorporación del Enfoque de Género, aflora también en sus paradojas, contradicciones y desencuentros internos que son el reflejo también, en ocasiones, de principios culturales que expresan la diferencia y que reivindican la autonomía de propuestas generadas, en muchas ocasiones, en el quehacer y la urgencia diaria. Los espacios de la desigualad son muchos, sus causas son múltiples y corresponden a situaciones muy diversas. Desde esta perspectiva aproximarse a escuchar otras voces es la mejor forma de contrastar y calibrar la propia realidad y los propios planteamientos, además de una base fundamental e imprescindible para comprender el rumbo de las reivindicaciones, los trabajos, las dificultades y, en última instancia, de los planteamientos que emanan de los propios actores sociales en sus contextos específicos.

Así pues, me ubico en el discurso característico de la antropología, el de la diversidad cultural, el que me permite, desde un oficio, unos conceptos y unos métodos ya contrastados, preguntarme por el qué, el cómo y el por qué de otras soluciones y de otras perspectivas vitales. Un planteamiento que me sitúa, de forma inmediata y necesaria, en la complejidad de las dinámicas sociales. Y desde este enfoque teórico y metodológico abordo el objetivo de mi análisis en los espacios de los Discursos de la Igualdad, utilizando los recursos de la Perspectiva de Género.

\section{Movimientos de mujeres indígenas}

Estas consideraciones son el contexto en el que se propone una aproximación inicial al trabajo e historia de la Coordinadora Nacional de Mujeres Indígenas de Panamá (CONAMUIP). Dicha aproximación no constituye un ejercicio de sistematización ya que no se plantea como una forma de explicar los procesos y las experiencias vividas ni tampoco como una reconstrucción, ordenamiento u organización de dichos procesos. Pero sobre todo, no es una sistematización porque, como afirma Mariela Arce (Arce 2001: 51) son las personas que han vivido la experiencia, las únicas que pueden saber qué valores y supuestos orientaron sus actuaciones y son ellas las únicas que pueden poner en marcha este proceso de revisión y análisis crítico encaminado a 
la mejora de sus prácticas futuras. Sin embargo, sí cabe pensar en el establecimiento de micro-alianzas que canalicen esfuerzos con objetivos concretos y definidos.

Comienzo por destacar la visibilización de su existencia, de su quehacer y de su proceso en la creencia de que es el más importante y prioritario de todos ellos. $\mathrm{La}$ visibilización es una reivindicación básica y clásica y una estrategia de primer nivel en múltiples procesos de sectores o grupos que están obligados a permanecer callados debido a su situación secundaria o subordinada frente a los sectores hegemónicos. No sólo porque dicha subordinación los ubique en la periferia, sino porque en muchas ocasiones estos sectores no logran ni siquiera ser vistos y oídos y por supuesto mucho menos percibidos (conocidos, comprendidos) y escuchados. Los grupos, sectores o individuos ocultos/ ocultados lo son, entre otras cosas, porque su realidad no es considerada importante, relevante o significativa, porque su posición social, más allá de sus condiciones de vida, es culturalmente secundaria y, desde luego, porque carecen de la capacidad de hacerse oír, lo que les impide convertirse en interlocutores de las instancias que detentan poder o algún tipo de poder. Estas consideraciones parten de la idea, ya planteada por Ardener (Moore 1991: 15) en su Teoría de los Grupos Silenciados, de que estos grupos, en realidad, son silenciados pero no silenciosos y que el problema radica en que permanecen ignorados, aún cuando pugnan por hacerse oír, porque no utilizan, porque no pueden utilizar los recursos y los espacios de transmisión y comunicación que les permitiría ser reconocidos. Los estudios de las mujeres y de género han considerado la propuesta de Ardener especialmente pertinente y adecuada porque permite destacar que, aunque la posición estructural de las mujeres ha sido y es mayoritariamente una posición subordinada ubicada en estructuras patriarcales, en realidad su voz no es que no haya existido, es que ha discurrido por otros cauces desde los que no era posible escucharla. En esta misma línea, Dolores Juliano planteó hace ya algunos años una visión que huía de cualquier posición victimista y defendía que, en realidad, las mujeres no habían sido nunca un sector pasivo de la sociedad y que, en muchas ocasiones, no asumían las ideologías y las practicas generadas para subordinarlas (Juliano 1992: 35). Esto implicaba que una de las vías de análisis más interesantes eran las que se orientaban al reconocimiento de las estrategias que habían desarrollado para subvertir esta situación. Son planteamientos que nos han ayudado a comprender que la invisibilidad es el resultado de la posición subordinada que las mujeres han ocupado en muchos lugares del mundo, pero no de su supuesta escasa responsabilidad en la estructura social y económica ni de su supuesto silencio. La incursión de los discursos de la igualdad de género habría supuesto una cierta quiebra discursiva de los sectores hegemónicos permitiendo que afloren unas voces que nunca estuvieron calladas y que se perciba el quehacer de las mujeres en la estructura económica y social. Así, en las estrategias y los procesos de visibilización, los sectores ocultos/ocultados se muestran dando a conocer sus pensamientos, enfoques, planteamientos, demandas, acciones y actuaciones, posibilitando la comprensión de su ubicación sociocultural, económica y política, permitiendo, en muchas ocasiones, reconocer su emergencia como actores sociales destacados (o que en algún momento ocupan papeles destacados) que actúan como referentes significativos. Se 
plantea que en muchas ocasiones estas estrategias de visibilización concluyen en situaciones de empoderamiento.

El relato que aquí se plantea parte de una microalianza surgida en Ciudad de Panamá entre la dirigencia de la CONAMUIP y la autora material de este artículo. El proceso de interlocución estableció una vía de intercambio de ideas que permitió la aproximación al conocimiento inicial de la realidad vivida por las mujeres que integran la Coordinadora Nacional de Mujeres Indígenas de Panamá, así como de sus procesos, planteamientos y luchas. Desde una perspectiva amplia, podemos ubicar a la CONAMUIP en el espacio de los Movimientos de Mujeres en América Latina, si por tales entendemos las diferentes propuestas, acciones y luchas desarrolladas en el continente americano por sectores de mujeres muy diversos a lo largo de las últimas cinco décadas. Estos movimientos han sido y son sumamente heterogéneos y responden a una casuística múltiple, derivada de los problemas específicos experimentados por las mujeres americanas en sus respectivos contextos históricos, políticos, económicos, sociales y culturales, por lo que sería necesario valorar, para su análisis, los procesos específicos que han generado cada uno de ellos. En realidad, el primer problema es la utilización del término Movimiento ya que bajo su paraguas se encuentran multitud de formas variadas de actividades políticas, acciones colectivas femeninas, modalidades asociativas o redes de solidaridad. En términos generales, se han elaborado numerosas tipologías en un intento por clarificar todas estas distintas formas de organización de las mujeres. En estas clasificaciones se hablaba de los movimientos de carácter feminista, ligados a la aparición de la segunda ola feminista, cuyas reivindicaciones se centraban en una modificación de la posición de las mujeres y por tanto respondían a intereses estratégicos de género. Otras formas características serían los movimientos por la supervivencia ligados a situaciones de pobreza, carestía y crisis económica, y los movimientos por los derechos humanos vinculados a crisis políticas, dictaduras y guerras. En ambos casos no se produciría una demanda de modificación de la posición de las mujeres, sino que responderían a intereses prácticos de género en función del desempeño tradicional de roles femeninos.

A esta clasificación clásica de formas organizativas hay que sumarles las formas específicas de organización de las mujeres indígenas, cuyos enfoques y perspectivas y cuyas reivindicaciones y demandas se formulan desde su autoidentificación étnica particular que incluye la visión desde su propio sistema de género histórico y presente. Para poder comprender todos los matices y riquezas de sus propios planteamientos es necesario, por tanto, centrar su ubicación en un ámbito más específico, el espacio de los Movimientos de Mujeres Indígenas que se ha configurado y se ha fortalecido en las dos últimas décadas. El empuje que los discursos de la igualdad han proporcionado a las demandas y acciones de múltiples actores sociales ha sido también un factor de impulso para las iniciativas de las mujeres indígenas y ha tenido como consecuencia la creación de diversas organizaciones, así como la formación de redes que han hecho oír sus voces y han hecho sentir su presencia para sugerir propuestas de acción en respuesta a la doble marginación que sufren, como mujeres y como indígenas. Algunas de sus consecuencias directas son la creación de espacios entre los que destaca, sin duda ninguna, la existencia del Enlace Continental de Mujeres 
Indígenas de las Américas-ECMIA y las múltiples iniciativas que de su seno han surgido. El Enlace Continental ha supuesto la articulación de las mujeres indígenas de América en una organización que representa a los pueblos indígenas de América agrupados en cuatro Enlaces regionales: El Enlace Norte (Canadá y Estados Unidos), el Enlace México (México y área Maya), el Enlace Centro (agrupa los países de Centroamérica) y el Enlace Sur (donde se encuentran agrupadas las mujeres indígenas de Venezuela, Colombia, Ecuador, Perú, Bolivia, Brasil, Argentina y Chile) ${ }^{4}$. El Enlace Continental surgió en 1993 a partir de la iniciativa de las mujeres indígenas canadienses quienes plantearon la necesidad de crear un espacio propio para las mujeres. En 1995 se realizaron una serie de talleres regionales que fueron la base del desarrollo posterior del Enlace Continental así como de los sucesivos Encuentros Continentales de Mujeres Indígenas que se han celebrado hasta el momento (Quito 1995; México 1997; Panamá 1999; Lima 2004; Quebec 2007), y de la convocatoria para la próxima celebración del VI Encuentro (México 2010). La visibilización de sus identidades, conocimientos y valores, a partir de su autoidentificación como pueblos indígenas junto con las críticas derivadas del desconocimiento de sus formas de vida, son reivindicaciones prioritarias en las declaraciones surgidas de los Encuentros Continentales, dirigidas a los gobiernos nacionales así como a organismos supranacionales.

Por otro lado, en este esfuerzo de contextualización, tampoco debemos olvidar que la emergencia de los movimientos de mujeres indígenas es un proceso que debe ser contemplado en un marco de emergencia y protagonismo de los pueblos indígenas a nivel mundial, tanto en los espacios transnacionales ${ }^{5}$ como en la concreción de proyectos políticos que han supuesto su participación a distintos niveles en espacios de poder locales y nacionales. Así, en un período de 20 años, hemos asistido a la visibilización y el protagonismo creciente de las voces de pueblos indígenas del continente americano y especialmente, para el caso que aquí nos ocupa, hemos escuchado cada vez con más fuerza los planteamientos desarrollados por las mujeres indígenas, facilitado por aspectos tales como las crecientes facilidades para el acceso a las nuevas tecnologías de la información y las comunicaciones que ha contribuido a la creación de redes y al intercambio de informaciones, promoviendo avances y cambios en la posición y desarrollo de los movimientos de mujeres indígenas. De la misma manera que el Movimiento de Mujeres de América Latina, considerado de forma global, se ha caracterizado históricamente por su heterogeneidad, también los movimientos de mujeres indígenas deben ser contemplados y escuchados en sus propios contextos políticos, históricos y económicos pero además, y sobre todo, sólo será posible comprender su posición, sus planteamientos, sus enfoques y demandas desde sus especificidades étnicas ligadas a sus pertenencias históricas.

4 Estos datos han sido elaborados por las siguientes organizaciones: Derechos y Democracia, Enlace Continental de Mujeres Indígenas, y Femmes autochtones du Québec (Mujeres Indígenas de Quebec). Pueden encontrarse en la referencia «Mujeres Indígenas de las Américas» en la página web de Derechos y Democracia.

5 Como referencia global hay que recordar que el Decenio Internacional de la Poblaciones Indígenas del Mundo fue proclamado por la Asamblea General de Naciones Unidas, el 21 de diciembre de 1993 para el período comprendido entre 1995 y 2004 cuya continuación se concretó en el Segundo Decenio que comenzó el 1 de enero de 2005. 


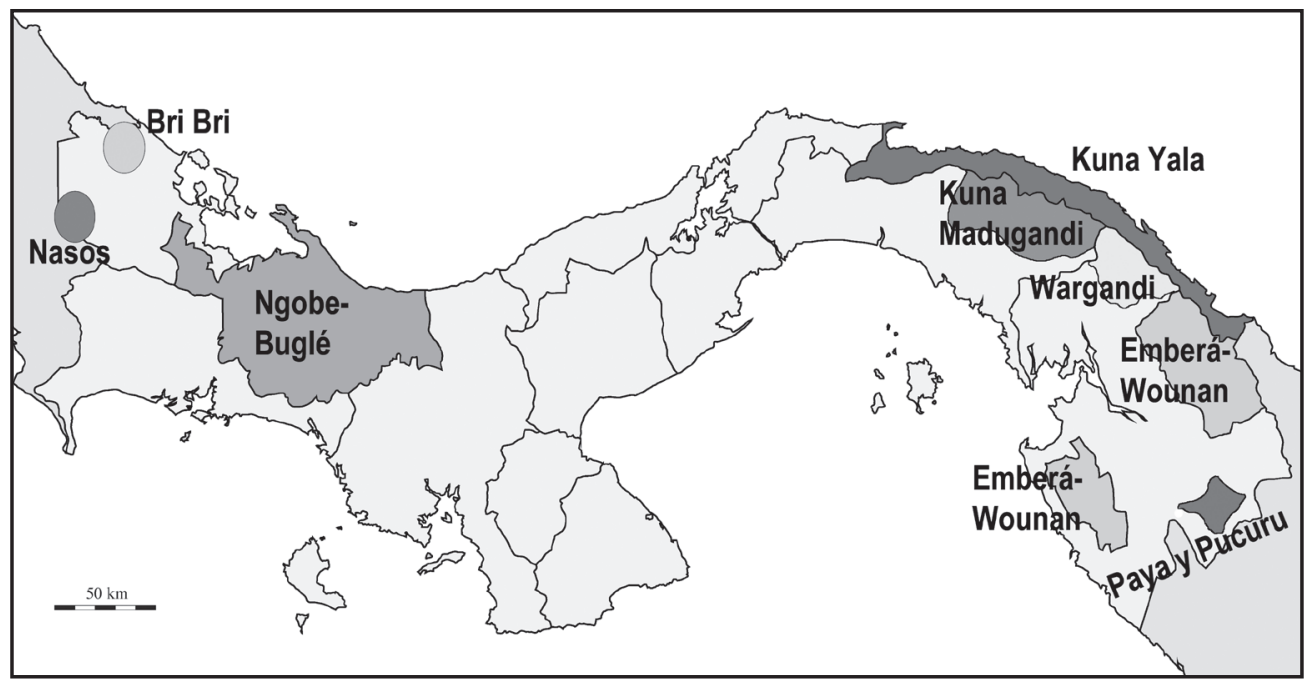

Figura 1: Mapa de Panamá con la localización de las comarcas indígenas

Para comprender el quehacer, evolución y significación de las organizaciones de mujeres en general, y de la CONAMUIP en particular, hay que tener en cuenta varios planos que nos permitan apreciar toda esa complejidad de la que hablamos. En primer lugar es necesario conocer su ubicación de partida, es decir identificar sus pertenencias culturales y su posiciones sociales y económicas en contextos históricos y políticos concretos. Después hay que conocer y comprender su evolución ligada a los propios esfuerzos desarrollados por generar, lograr y aceptar presencias en foros múltiples donde visibilizan sus planteamientos, objetivos, metas. Es necesario, además, comprender los contextos que conforman escenarios poliédricos con imbricaciones y relaciones con multiplicidad de actores. Escenario y espacios donde su presencia es demandada y en los que se convierten en auténticos referentes de los pueblos indígenas en su conjunto. Todo ello genera cambios en las actitudes, las actuaciones y los discursos que dan cuenta de los éxitos y las quiebras de todo el proceso. El camino transitado tiene consecuencias diversas, de tal manera que a lo largo del tiempo es posible evaluar la capacidad generada de negociar y de influir en organizaciones e instituciones de distinto nivel (local, nacional, global) incluyendo demandas específicas de la organización. También, inevitablemente, sus actuaciones producen cambios en el seno de la propia organización y es posible apreciar historias de empoderamiento individual y colectivo.

\section{Panamá y la CONAMUIP}

La República de Panamá se encuentra situada en el Itsmo de Panamá, en América Central, bordeada en su parte norte por el Mar Caribe y en su parte sur por el Océano 
Pacífico y con fronteras este y oeste, respectivamente, con Colombia y Costa Rica. La referencia a su ubicación geográfica es especialmente importante ya que esta privilegiada situación ha jugado un papel decisivo en su desarrollo histórico y en los procesos políticos, económicos y sociales que han tenido lugar en su territorio. Así, no es posible comprender la historia contemporánea de Panamá si nó se alude a todos los acontecimientos sucedidos en torno a la construcción, control y gobierno del Canal Interoceánico. Panamá está dividida administrativamente en nueve provincias, 3 comarcas indígenas (Kuna Yala, Emberá, Ngöbe-Buglé), que tienen nivel de provincia, y 2 comarcas (Kuna de Madungandí y Kuna de Wargandí) con nivel de corregimiento (Figura 1). En el conjunto de estos territorios se distribuye una población que según el avance de datos de los Censos Nacionales 2010 asciende a 3.322 .576 personas ${ }^{6}$.

La población indígena (cuya ubicación en el territorio panameño está reflejada en la Figura 1), representa aproximadamente el $10 \%$ del total de población constituida por siete pueblos claramente definidos: kuna, emberá, wounaan, ngobe, buglé, nasoy bri-bri, ubicados a lo largo de todo el territorio panameño, tanto en las propias Comarcas Indígenas como en los centros urbanos, especialmente en Ciudad de Panamá, la capital de la República, donde confluye un significativo flujo migratorio.

Es en Ciudad de Panamá donde tiene su sede CONAMUIP, la Coordinadora Nacional de Mujeres Indígenas de Panamá, una organización que aglutina y representa al conjunto de las mujeres indígenas de los pueblos indígenas de Panamá. En un contexto en el que las reivindicaciones indígenas han estado muy ligadas a las demandas específicas realizadas desde cada uno de los pueblos presentes en Panamá, este carácter aglutinador es especialmente relevante. De esta manera la CONAMUIP se presenta como una organización indígena panameña interétnica que pone especial énfasis en la participación y colaboración explícita y permanente entre los diferentes pueblos que la componen. Esto no significa, sin embargo, que sea la única organización de mujeres indígenas de Panamá. Es necesario destacar también la existencia de la Asociación de Mujeres Ngäbe-Buglé (ASMUNG) que agrupa a mujeres ngobe de diferentes comunidades de la comarca Ngobe-Bugle ${ }^{7}$.

La aproximación al conocimiento político, científico y vital de la Coordinadora se puede producir por varias vías. Detenernos un momento a reflexionar sobre estos caminos no es solamente una concesión a la metodología desde perspectivas académicas sino que forma parte del proceso más amplio de aprehensión de las vidas, esfuerzos y caminos transitados por las mujeres que protagonizan sus actuaciones, individual y colectivamente. Un primer camino de visibilización directa e inmediata es acceder a su propia página $\mathrm{web}^{8}$ donde nos hablan de su historia y estructura, nos indican sus objetivos y dan cuenta de las actividades que realizan, los eventos en los que intervienen y a los que son llamadas y los proyectos en los que participan. Incidir en este aspecto supone dar cuenta de la importancia de generar espacios propios de

\footnotetext{
6 Datos de la Dirección de Estadística y Censo de la Contraloría General de la República.

7 De hecho, la Asociación de Mujeres Ngäbe-Buglé se constituyó algún tiempo antes que CONAMUIP (1991). Es posible consultar sus actividades en la página web de ASMUNG.

8 Coordinadora Nacional de Mujeres Indígenas de Panamá (CONAMUIP).
} 
carácter globalizado que les permiten de alguna manera controlar sus propias definiciones y presentaciones a través de las nuevas tecnologías de la información. Así pues, llamar la atención sobre estos procedimientos es una forma de reconocimiento de los esfuerzos realizados por hacerse visibles y ser ubicadas en su papel de agentes sociales activos, teniendo en cuenta que su consideración como referentes en el contexto de pueblos indígenas es cada vez mayor, tanto en el interior del país como en los espacios internacionales.

En esta línea de identificación de itinerarios de la propia organización, es necesario trabajar con atención los referentes bibliográficos generados por ellas mismas y aquellos que reflejan sus actuaciones en múltiples eventos colectivos, muchos de ellos ligados a las actividades realizadas a lo largo de casi dos décadas por el Enlace Continental de Mujeres Indígenas de las Américas-ECMIA y desde luego, las relaciones mantenidas con otros movimientos de mujeres, en el interior de Panamá, especialmente su participación en la Alianza del Movimiento de Mujeres de Panamá así como en el Consejo Nacional de la Mujer de la que CONAMUIP es parte. Pero, sin duda, escuchar de forma directa su propio relato ${ }^{9}$ es la mejor forma de poder aproximarse al conocimiento de los procesos de formación y mantenimiento de una organización de mujeres que se inscribe en el contexto de los Movimientos de $\mathrm{Mu}-$ jeres en América Latina y específicamente protagoniza los movimientos de mujeres indígenas en Panamá.

En primer lugar, hay que decir que la aparición de la CONAMUIP no puede desligarse del proceso de fortalecimiento del Movimiento de Mujeres de Panamá a principio de la década de los 90 (después de la invasión de Panamá por el ejército de los Estados Unidos), así como de las iniciativas institucionales surgidas a lo largo de la década. Entre las muchas iniciativas que han tenido lugar desde ese momento hasta la actualidad, cabe destacar la creación y posterior fortalecimiento del Consejo Nacional de la Mujer (CONAMU) y la Dirección Nacional de la Mujer (DINAMU), adscritos en primera instancia al Ministerio de Trabajo y Bienestar Social, posteriormente al recién creado Ministerio de la Juventud, la Mujer, la Niñez y la Familia, y actualmente al Ministerio de Desarrollo Social. Ambas instituciones son concebidas como espacios y mecanismos institucionales orientados a fomentar y apoyar procesos a favor de la igualdad de género, entre las que se cuenta la elaboración y publicación del Informe Nacional Clara González: Situación de la Mujer en Panamá, cuya última edición (el IV Informe 2008), abarca el período 2002-2007. El apartado $8.2 \mathrm{se}$ dedica especialmente a «Mujeres indígenas: de la invisibilidad a la participación con equidad». En este apartado se citan los instrumentos internacionales de protección de los derechos humanos. Aunque entre ellos se cita al Convenio 169 sobre Pueblos Indígenas y Tribales de la OIT, hay que decir que su no ratificación hasta el momento actual por parte del Estado panameño es uno de los caballos de batalla de los pueblos indígenas panameños y uno de los objetivos, por tanto, de las mujeres indígenas panameñas.

9 Ello fue posible gracias a la ayuda ofrecida por Sonia Henríquez y Ebinia Santos. 
Como relata Mariela Arce (Arce 2001: 54), es en ese momento cuando se constituye la alianza denominada Foro Mujer y Desarrollo que puso en marcha la elaboración del Plan Mujer y Desarrollo (1994-2000). En el mismo participaron cerca de 50 organizaciones que realizaron un diagnóstico de la situación de las mujeres panameñas y diseñaron las líneas de actuación para la puesta en marcha del mismo. Sus actividades estuvieron apoyadas por el Programa Promoción de la Igualdad de Oportunidades en Panamá (PROIGUALDAD), resultado del acuerdo entre el Movimiento de Mujeres y el Estado panameño. Su objetivo era la ejecución del Primer Plan Nacional Mujer y Desarrollo para lo que se procedió a la firma de un Convenio de Financiación entre la Unión Europea y la Republica de Panamá (1996) para el período comprendido entre 1997 y 2002.

Paralelamente, en el año 1993, un grupo de mujeres indígenas que venían de diferentes procesos organizativos, como nos relata Sonia Henríquez ${ }^{10}$, reciben una invitación para participar en el Segundo Encuentro Nacional de Mujeres Panameñas, en el Colegio Javier de la Ciudad de Panamá. Es el espacio de encuentro no sólo con otros movimientos que formaban el Comité Nacional de Mujeres Clara González, sino que es, sobre todo, el lugar de encuentro con las mujeres de otros pueblos indígenas de Panamá. De este encuentro surge la iniciativa de crear una instancia, un espacio común en el que las mujeres indígenas pudieran plantear sus objetivos y propósitos comunes con el fin de fortalecer las organizaciones comunitarias de sus comarcas y comunidades. La idea es poder ser un aporte como mujeres indígenas hacia su sociedad, hacia los pueblos indígenas en un trabajo conjunto con las autoridades tradicionales. De ahí que se piensa fundamentalmente en lo local, sin tener muy presente, en ese momento lo nacional y lo internacional.

En este primer momento no están presentes los 7 pueblos indígenas ubicados en el territorio de la República de Panamá, sino que son las mujeres de los tres grupos indígenas mayoritarios de Panamá ${ }^{11}$ : kuna, emberá-wounaan y ngobe-buglé, las que van a comenzar a organizarse a través de la formación de sus propias coordinadoras: Organización Comarcal de Mujeres Kunas Olowaili, Coordinadora Comarcal de Mujeres Emberá-Wounaan y la Coordinadora General de Mujeres Ngobe-Buglé. De esta manera, se dan los primeros pasos para poner en marcha una organización con afán de representar al conjunto de las mujeres indígenas panameñas, tanto a las mujeres residentes en sus comunidades como a aquellas que se han trasladado a zonas urbanas. De hecho, la Coordinadora se estructura en sectoriales, siendo el Sector 5 el que se ocupa de las áreas urbanas de Colón y las barriadas establecidas en torno a Ciudad de Panamá.

Así pues, la Coordinadora Nacional nace en 1993, en el Primer Encuentro Nacional de Mujeres Indígenas celebrado en Capira. Será posteriormente, en 1999, cuando logren su personería jurídica, no sin algunos temores y reticencias ya que se asimilaba la obtención de la personería jurídica con las formas de organización de las ONGs, lo que desvirtuaba, desde su punto de vista, las bases de constitución y funcionamiento de la Coordinadora:

\footnotetext{
10 Entrevista con Sonia Henríquez.

11 Estos datos pueden ser consultados en la página web de la Coordinadora.
} 
«Al inicio fue un poco difícil, porque siempre como Coordinadora nosotras nos reuníamos y decíamos: la Coordinadora Nacional no puede ser una ONG. Nosotras lo veíamos que tener una personería jurídica es como poner ese sombrero de una ONG y ese no era el objetivo de la creación porque el objetivo es que toda mujer indígena es parte de CONAMUIP, por eso no podemos hablar cuántas somos..., quiénes son las miembras de CONAMUIP, no lo podemos hablar. Cuando una ONG se crea tiene que tener 25 personas para que tengas tu propia personería jurídica, o de la noche a la mañana de repente las personas se reúnen y dicen vamos a crear una ONG, esa no es la creación de la Coordinadora Nacional. La Coordinadora Nacional se crea dentro de los pueblos indígenas de modo que toda mujer es parte de la Coordinadora Nacional, activa, pasiva, lo que sea pero es miembra de la Coordinadora Nacional. Yo pienso que esa es la diferencia que nos hace sentir con las otras ONGs, digamos organizaciones» (Sonia Henríquez).

En este comienzo todavía no existe ningún proyecto concreto que articule su trabajo sino que los esfuerzos se centran en poder trabajar desde su propio liderazgo (Pizarro 2001: 16) para contribuir al avance de los pueblos indígenas teniendo, además, siempre presente la búsqueda de la colaboración y aceptación de sus iniciativas $\mathrm{y}$ actividades por parte de las autoridades indígenas. Este es un principio que debe ser entendido en relación con las propias formas de organización política de los pueblos indígenas de Panamá que están estructuradas mayoritariamente en torno a los denominados Congresos Generales ${ }^{12}$, además de otras estructuras políticas particulares ${ }^{13}$. Su perspectiva específica se registra en las palabras de Sonia Henríquez cuando afirma que

«Siendo una mujer indígena siempre vamos a ver lo que es la parte de la colectividad, nunca nos vamos a ver solamente como mujer. Siempre en nuestros temas, en nuestras agendas se va a ver los pueblos indígenas. Vamos a ver la problemática que estamos viviendo dentro de nuestras comunidades. Esa es la particularidad en la que siempre nos hemos manejado y es lo que hemos dicho como mujeres indígenas».

Son, por tanto, su pertenencia étnica y su identidad cultural, la defensa de los valores tradicionales, además de su condición de mujeres, las bases fundamentales sobre la que se sustenta la creación y consolidación de la CONAMUIP así como los principios que rigen las orientaciones y directrices que van a guiar sus pasos. Estos planteamientos tienen que ver, por tanto, con las estructuras y formas de organización políticas específicas de cada uno de los pueblos indígenas pero además, y esto es muy importante, van ligados a la propia concepción de los sistemas de género propios de cada pueblo. En este aspecto su perspectiva pivota, al menos teóricamente, sobre la concepción de la dualidad y de la complementariedad que presupone la posibilidad de poder trabajar con el compañero hombro con hombro, de poder realizar una tarea común. Un argumento basado en referencias concretas, en los que se resalta, en el caso del pueblo kuna, la importancia del nacimiento de la niña o su papel relevante

12 Congreso General Kuna; Congreso General de la Comarca Emberá; Congreso General de la Comarca Kuna de Madungandi,; el Congreso General de la Comarca Ngöbe-Buglé y el Congreso General de la Comarca Kuna de Wargandi.

13 Es el caso de la monarquía naso. 
ligado a una descendencia de carácter matrilineal y se recuerda como, desde los cantos tradicionales y desde su propia historia, ellas saben que la mujer ha jugado y juega un papel importante dentro de la sociedad indígena.

Desde una perspectiva externa a su propia realidad destaco como, en relación con la posición que ocupan las mujeres en el seno de sus propias culturas, esta es una concepción percibida como contradictoria en su propio funcionamiento interno y semejante a otros discursos culturales de otros pueblos en América Latina. Estos argumentos defienden la existencia de sistemas de género en los que los estereotipos femenino y masculino tienen atribuciones de carácter complementario: cada uno posee aquello de lo que el otro carece. Eso significaría una dualidad que los hace necesarios mutuamente y que establece unos sistemas de reciprocidad complementaria basados en unas relaciones de género equitativas. Su existencia supondría la supervivencia de normas culturales prehispánicas que se habrían mantenido más allá de la imposición de los sistemas de género patriarcales que la conquista y colonización implantan y que continúan vigentes hasta el momento actual.

Sin embargo, y de la misma manera que también en nuestras propias sociedades crece el número de personas que, en un argumento de ida y vuelta, asumimos el contraste paradójicamente cada vez más evidente entre la igualdad formal (en el caso de tratarse de lugares donde la igualdad formal se haya logrado) y la igualdad real, también en este caso se observa una divergencia entre los principios culturales mantenidos como ideales y que actúan como referentes históricos y los comportamientos reales. Así, los principios culturales ligados a la complementariedad y a las concepciones dualistas en cuanto a las relaciones de género, que supondría, al menos teóricamente, la existencia de un sistema equitativo en el que las mujeres no ocuparían una posición subordinada, es percibido desde las propias organizaciones como principios debilitados, en el mejor de los casos. Así, se habla de la complementariedad que debe existir pero se reconoce que hay otra realidad diferente a la que está en la «propia historia». Esto habría tenido como consecuencia una mayor pasividad en las mujeres ligada a las dificultades o imposibilidades para defender sus posiciones $\mathrm{y}$, desde luego, para poder estar en la toma de decisiones. También en este caso, aunque de muy diferente manera y por muy diferentes causas, las mujeres indígenas de Panamá vivirían la dicotomía entre igualdad formal histórica e igualdad real como una de las realidades contradictorias surgidas de las identidades múltiples.

Por un lado la variable étnica es básica en los planteamientos de reivindicación y lucha, es la matriz de la cual se parte en un enfoque que también está ligado en muchas ocasiones a las demandas surgidas de las situaciones de carencia y pobreza. Desde la Coordinadora se tiene siempre presente que la base de sus trabajos está en la identidad cultural y, por tanto, es necesario partir de las características que definen las peculiaridades de los diferentes pueblos que conforman la Coordinadora. Así, es posible decir que la fuerza y la particularidad de trabajo de las kunas deriva de la importancia concedida a su propia identidad cultural; las mujeres ngobe, en razón de su propia historia, son especialistas en las estrategias de comercialización de sus propios trabajos artísticos/artesanos mientras que las mujeres emberá tienen en común sus actividades de carácter comercial al frente de cooperativas y de tiendas comunitarias. 
Existe, por tanto, un reconocimiento basado en el respeto a las diferencias históricas y culturales de las integrantes de la Coordinadora que según sus principios, no lo olvidemos, son todas las mujeres indígenas de Panamá ${ }^{14}$. Sin embargo, la Coordinadora no sólo responde a las demandas como pueblos indígenas sino que es claramente una organización de mujeres con plena autoidentificación como tales, con estrategias derivadas de su posición en la producción y la reproducción expresando la pluralidad de experiencias presentes en las identidades de género.

«Pero además de la parte de lo colectivo, tenemos muchas cosas en común, porque si no la coordinadora no existiría. Siento que en toda la organización, en el proceso que hemos tenido hay mucho la parte de la sensibilidad, eso es lo que nos une como mujeres, como madres, también como lideresas, siempre viendo hacia nuestras comunidades, viendo hacia las indígenas, la juventud, la niñez, yo siento que esa ha sido la fuerza que nos ha unido por la que tenemos tantos años nuestra presencia» (Sonia Henríquez).

Lo que se produce a lo largo de los años de existencia de la Coordinadora Nacional es una evolución donde se va materializando un discurso propio gestado en y desde la propia organización, pero que no es ajeno, no puede serlo, a los contactos y las relaciones mantenidas con otros movimientos sociales y con otras instancias que son un apoyo relevante en cada una de las acciones que realizan. Esto supone contemplar las aportaciones de carácter teórico y metodológico que incorporan a su propio discurso así como el carácter de estas relaciones con diferentes actores sociales. El proceso ha estado y está ligado a procesos de formación y de elaboración de sus propios productos de carácter teórico, algunos de ellos elaborados como guías ${ }^{15}$ para el desarrollo de talleres educativos que promuevan las participación de todas las mujeres y que son una de las iniciativas más importantes para la Coordinadora. Así, en estas guías elaboradas por la propia CONAMUIP y apoyadas por Proigualdad, algunos de los temas centrales giran en torno a la organización, la planificación y el fortalecimiento del proceso organizativo; la violencia intrafamiliar; la autoestima o la comercialización para mujeres indígenas desde la perspectiva de género. Cabe destacar, en la línea planteada sobre la influencia e impacto, a todos los niveles, de los Discursos de la Igualdad, la incorporación de la Perspectiva de Género como un útil instrumento de análisis ${ }^{16}$. Así, siguiendo sus propios planteamientos, vemos que la distinción entre sexo y género, la diferencia entre lo biológico y lo cultural, permite afirmar que la percepción que se tiene de la mujer deriva de las concepciones sexistas del mundo occidental, culturales por tanto, donde no se reconoce la capacidad de las mujeres. Una afirmación en sintonía con las consecuencias de la traslación del sistema patriarcal español en la conquista.

14 Sin olvidar la existencia de la Asociación de Mujeres Ngäbe-Buglé (ASMUNG).

15 Un ejemplo son las guías didácticas denominadas Unidos Tejemos Nuestro Futuro que son a su vez el resultado de las aportaciones hechas por las propias mujeres en diferentes seminarios y grupos de trabajo. Agradezco a la Coordinadora haberme permitido su consulta.

16 El IV Informe Nacional «Clara González» dice que «la protección de los derechos humanos de las mujeres indígenas, es un logro que ha emanado al amparo de las grandes luchas del movimiento de mujeres traducidas en leyes con enfoque de género «extensivas» a las mujeres indígenas» (p. 183). 
En la línea de comprender los significados de la incorporación de esta perspectiva, hay que decir en primer lugar que la noción de género y sus conceptos asociados (relaciones de género, identidad de género, estereotipos de género, roles de género, intereses de género, etc.), es decir, todo lo que define y nos permite analizar los sistemas sociales como sistemas de género son, en su conjunto, un producto de carácter teórico elaborado en las últimas décadas por intelectuales feministas occidentales que ha traspasado los límites académicos y ha permeado los planteamientos institucionales a nivel local, nacional e internacional. En este proceso, sabemos que su desarrollo ha sido tan amplio que forma parte de las bases teóricas de múltiples diseños políticos, de la elaboración de normas legislativas, de los programas de cooperación y ayuda al desarrollo o/y de los desarrollos curriculares de estudios de diferente nivel en escuelas y universidades, entre otros ámbitos de aplicación. El término Género se ha incorporado mayoritariamente al lenguaje de organismos de diferentes niveles, locales, nacionales o transnacionales, incluyendo la reciente creación de la Entidad de las Naciones Unidas para la Igualdad entre los Géneros y el Empoderamiento de la Mujer conocida como ONU Mujeres ${ }^{17}$. Sin embargo, la Perspectiva de Género no ha tenido un enfoque unidireccional y mucho menos un desarrollo unitario en sus concepciones, bien al contrario su evolución ha hecho de él un discurso rico y heterogéneo basado también en su producción multidisciplinar, en su aplicación variada $\mathrm{y}$ en los diferentes sujetos y sectores sociales que lo han adoptado como herramienta de análisis y diseño. De la misma manera que los enfoques feministas también son diversos y han incorporado la producción de feminismos procedentes de otras áreas del mundo que han cuestionado las relaciones colonialistas en todos los ámbitos incluyendo el colonialismo discursivo del feminismo blanco occidental, entendido como una relación de poder entre distintos saberes, su incorporación a los planteamientos teóricos de la CONAMUIP habla de otras perspectivas orientada a sus intereses y necesidades específicas. Esta sería una de las formas de concreción práctica de una de las reivindicaciones defendidas por los denominados feminismos poscoloniales es decir, la necesidad de reconocer el lugar desde donde se habla, el contexto histórico y social desde donde se percibe la realidad para poder comprender las complejidades y contradicciones que marcan las identidades sociales, muchas veces ubicadas en cruces de fronteras conformando lo que han denominado identidades de frontera (Hernández 2008: 80).

Naturalmente la utilización de la Perspectiva de Género no significa, en absoluto, la adopción de una perspectiva feminista que, por otro lado, está alejada de sus propios planteamientos pero sí nos habla de esa influencia de los discursos de la igualdad a la que nos referíamos y también de las herramientas metodológicas utilizadas por muchos de los interlocutores que las organizaciones de mujeres, en general, y la CONAMUIP, en particular, tienen en el conjunto de sus actividades. También da cuenta de la evolución experimentada, de las nuevas posiciones que se van adoptando y de las aportaciones para los propios movimientos que los Enfoques de Género apor-

17 La misma necesidad de seguir poniendo en marcha estas iniciativas demuestra que el objetivo se encuentra todavía muy lejano y que, además, en muchas ocasiones, la mayoría de las acciones se limitan a generar espacios de mujeres para mujeres sin conseguir cambios sistémicos significativos. 
tan en su quehacer. Muchas de estas actividades se realizan en colaboración y con el apoyo directo de organismos nacionales e internacionales (Unicef, Oit, Onusida, ONGs nacionales e internacionales) cuyo apoyo permite los trabajos de planificación y autoevaluación de la propia Coordinadora así como la puesta en marcha de diferentes proyectos (entre ellos la elaboración de materiales que definen sus principales demandas: la identidad y la promoción del liderazgo de las mujeres, el fortalecimiento institucional de las organizaciones, los problemas derivados del Vih/Sida y sus consecuencias en las mujeres indígenas de Panamá, Convenio 169 de la OIT, etc.). En este sentido, las mujeres de la Coordinadora destacan el hecho de que la Coordinadora Nacional es una organización integral ya que, como instancia nacional, tienen que velar por todo lo que tiene que ver con los derechos humanos de las mujeres y con los problemas sociales presentes en las comunidades indígenas. La CONAMUIP se autodefine, por tanto, como una organización macro en la que se trabajan todos los temas que afectan a los pueblos indígenas y especialmente a las mujeres indígenas (interculturalidad ${ }^{18}$, violencia, la erradicación del trabajo infantil, salud, drogas y Vih/ Sida, conocimientos tradicionales de los pueblos indígenas, el medio ambiente, acciones encaminadas al fortalecimiento individual o colectivo, organización comunitaria, elaboración de proyectos, liderazgo, autoestima, etc.). Cabe destacar el diseño y la elaboración reciente del CD Interactivo de los Pueblos Indígenas de Panamá ${ }^{19}$ en colaboración con Unicef y la Agencia Española de Cooperación Internacional para el Desarrollo (AECID) que está concebido como un material de apoyo para promover el conocimiento de los pueblos indígenas de Panamá y cuyo objetivo es ser difundido por los educadores en los establecimientos escolares. La importancia de toda esta producción no sólo radica en su propio valor sino que es un conjunto de valiosos instrumentos que aportan sugerencias metodológicas para las facilitadoras que conducen las reuniones y talleres en las comarcas y comunidades.

«También siento que hay mucha capacidad, mucha fortaleza en recursos humanos, la capacidad de dominio de temas. De aquí han salido facilitadoras. Nosotras mismas somos las facilitadoras cuando vamos a nuestras comunidades. No necesitamos de personas no indígenas que sean facilitadoras en nuestras comarcas porque nosotras como mujeres indígenas hay capacidad en esta organización. Esa ha sido también nuestra lucha de dar ante otra gente: que nosotras también podemos, tenemos conocimientos y es una riqueza de conocimientos porque el llevar un tema lo vamos a hacer desde nuestro sentir, porque es diferente hablar de autoestima que una persona no indígena nos venga hablar que lo pueda hacer una mujer indígena» (Sonia Henríquez).

Todas estas referencias teóricas son parte de los contextos que conforman esos diferentes escenarios a los que me he referido. Todo ello canalizado a través de los mecanismos de funcionamiento interno que suponen establecer vías de comunicación

18 En palabras de Ebinia Santos la interculturalidad se plantea de la siguiente manera: «respetar los derechos de todos los pueblos. Yo como ngobe voy al pueblo kuna y debo respetarlos y aceptarlos como son y ellos viceversa, es una convivencia entre todos los pueblos» (entrevista con la autora).

19 Elaborado en sus contenidos por Sonia Henríquez (pueblo kuna), Eladia Carpintero y Mary Acosta (pueblos ngäbe-buglé), Floricelda Peña (pueblos emberá wounaan) y Bernal Castillo (pueblos naso y bri-bri) Es posible consultarlo en el Portal Educativo «Educa Panamá» del Ministerio de Educación de Panamá. 
entre las bases de la organización. De esta manera, internamente, la CONAMUIP promueve una relación permanente entre las mujeres indígenas residentes en las comarcas, las comunidades y las barriadas indígenas, a través de los engranajes de los que se ha dotado: las Coordinadoras Comarcale ${ }^{20}$ y representantes de cada uno de los pueblos. De nuevo se resalta su condición de espacio intercultural en el que comparten sus experiencias a partir de sus problemas comunes y de los proyectos en los que colaboran y participan.

«Yo le puedo hablar de la comarca emberá porque lo he visto, he estado dentro, he estado con las mujeres emberá, he compartido con las mujeres emberá, he compartido su techo, he estado en su comunidad. De igual el hablar de las mujeres ngobe, igual manera, con las mujeres kuna, con las mujeres naso, con las mujeres bri-bri. De igual las mujeres ngobe han estado en la comarca Kuna-yala. De igual las emberá han estado con las ngobe, con las kuna. Por eso esta CONAMUIP cuando sale en representación de alguien afuera o en nacional, uno puede, teniendo el sombrero de la CONAMUIP, hablar y decir que es lo que queremos para o con las mujeres ngobe, para las mujeres emberá, eso ha sido una fuerza para nosotras» (Sonia Henríquez).

Más allá de su organización interna, la CONAMUIP establece relaciones en una múltiple vía que puede contemplarse como una red desde su propia posición central. De tal manera que como Coordinadora Nacional de Mujeres Indígenas de Panamá está en contacto con las autoridades originarias de cada uno de los pueblos indígenas de Panamá. También mantienen una presencia activa en los diferentes organismos nacionales a los que pertenecen y donde tienen una participación efectiva, como en el caso de la creación del Instituto de la Mujer de Panamá (INAMU), o en la elaboración de un conjunto de leyes en representación de las mujeres indígenas ${ }^{21}$. Por otro lado cabe destacar las relaciones establecidas con otros movimientos de mujeres a nivel nacional e internacional ${ }^{22}$. La CONAMUIP siempre ha tenido una presencia activa en ámbitos internacionales haciéndose presente allá donde ha sido convocada y requerida y donde y cuando ha tenido la oportunidad de hacerlo. Hemos nombrado anteriormente la importancia del Enlace Continental de Mujeres Indígenas y el papel destacado que la Coordinadora ha desempeñado en su desarrollo. Como ejemplo destacado de ello es necesario citar la celebración del Tercer Encuentro Continental de Mujeres Indígenas que tuvo lugar en Panamá, en el mes de marzo del año 2000. Previamente, en 1999, el Enlace crea la Secretaría de Comunicación y, dado que el Tercer Encuentro se va a realizar en Panamá, decide que sea la CONAMUIP quien asuma esta responsabilidad en un momento de construcción y avance del propio

20 Las Coordinadoras Comarcales promueven Encuentros Comarcales periódicos donde se realizan las tareas de evaluación y diagnóstico y se formulan propuestas.

21 Ley N 4 de 1999, por la cual se instituye la igualdad de oportunidades para las mujeres; Ley 20 del 26 de junio del 2000, que protegen el Derecho Colectivo de Propiedad Intelectual y Conocimientos Tradicionales; Ley $N^{\circ} 3$ del 5 de enero de 2000 sobre las Infecciones de Transmisión Sexual, el virus de la Inmunodeficiencia Humana y el SIDA; Ley $N^{\circ} 38$ del 10 de junio de 2001 que reforma y adiciona artículos al Código Penal y Judicial sobre violencia doméstica y maltrato al niño, niña, adolescente.

22 Es posible consultar en su página web, los Encuentros de Mujeres indígenas celebrados, así como los foros y Talleres organizados y los eventos internacionales en los que han participado. 
movimiento ${ }^{23}$. Del 5 al 8 de marzo de 2000 se reunieron en Ciudad de Panamá 70 mujeres indígenas de Abya-Yala ${ }^{24}$ para debatir sobre las diferentes formas de discriminación que afectan a las mujeres indígenas. Sus conclusiones se recogieron en la Declaración de Olowaili ${ }^{25}$.

En este contraste con otros movimientos se visibiliza la gestación y consolidación de la CONAMUIP como un espacio político, un espacio de reconocimiento, que permite que las voces y la presencia de las mujeres indígenas puedan expresar sus demandas aunque, en muchas ocasiones, en espacios políticos restringidos también por ser específicamente femeninos.

«A veces las mujeres no indígenas te dicen por qué tenemos que poner las mujeres indígenas, porque tiene que haber un artículo especificando para ellas si todas somos iguales, si todas somos mujeres, ahí es de donde hemos sentido que ha valido la organización nacional, que es un espacio para nosotras de poder hacer también nuestras demandas, nosotras siempre hemos dicho: sí, somos mujeres pero quiera o no quiera esas mujeres indígenas tienen su propia particularidad, tienen su propia identidad que no es igual que todas. El estar en un movimiento, si dicen que todas somos iguales, si yo le hablara mi idioma a las que están ahí no me van a entender, de ahí es donde se menciona la particularidad que existe, de una manera u otra hay una diferencia. Yo pienso que ese espacio también nos ha servido para eso, ha sido también una educación para la población general, para las mujeres que no son indígenas» (Sonia Henríquez).

Sin embargo, también es necesario decir que la creación y consolidación de un espacio como el que en estos momentos ocupa la CONAMUIP, es un camino lleno de dificultades y obstáculos. Así, es posible hablar de éxitos y también de quiebras y de debilidades. Por un lado, podemos hablar de un contexto que acoge y refuerza sus posiciones dentro de la emergencia política de los pueblos indígenas a nivel continental y de la pertinencia institucional del discurso de género que incluye los movimientos de mujeres. Así, se convierten en referentes de los pueblos indígenas a nivel nacional, su presencia es demandada por organismos nacionales y supranacionales y su colaboración es solicitada en diferentes acontecimientos, de la misma manera que se requiere su participación en diferentes proyectos que incluyen necesariamente la participación de las mujeres. Como también se convierten en imagen de los pueblos indígenas (me pregunto si también de Panamá) a través de la utilización de recursos materiales y especialmente del vestido, que de ser un elemento de discriminación y rechazo pasa a ser utilizado como un arma política de gran eficacia ${ }^{26}$. Pero sin duda

23 Hay que pensar que muchas de las organizaciones que formaban parte del Enlace Continental tenían serias dificultades para su participación incluyendo los problemas de comunicación. Algunas organizaciones no tenían acceso al correo electrónico y las informaciones a veces ni siquiera llegaban.

24 Nombre por el que el pueblo kuna denomina al continente americano y cuya utilización se ha generalizado.

25 Olowaili es el símbolo de la lucha de la mujer kuna. Es posible consultar la Declaración de Olowaili en la página web de Derechos y Democracia (Canadá).

26 El vestido es un símbolo de pertenencia étnica con significados polisémicos. Así, en una conversación mantenida con Sonia Henríquez y Ebinia Santos se planteaba que mientras para las kuna, la mola (su vestido tradicional) es la imagen de su identidad, es símbolo de orgullo y de dignidad, para las ngobe el vestido representa cosas distintas y se asocia a la pobreza y al pasado colonial. Además de los significados específicos, también hay que tener en cuenta que el vestido tradicional ha sido motivo de discriminación, desprecio e incluso de veto para muchas mujeres que han debido reivindicar con empuje y persistencia la utilización de sus 
estos elementos, que se pueden considerar ventajosos, pueden incidir en la aceleración de los procesos y en dificultar la construcción de una estructura organizativa, administrativa y de gestión que tenga la capacidad de asumir todos los retos que se les presentan. Entre las carencias detectadas y las demandas consiguientes se encuentran siempre presentes las vías de financiación aunque, en estos momentos, uno de los mayores problemas deriva, probablemente, del alto nivel de voluntarismo de las mujeres más implicadas en la dirigencia de la Coordinadora que dificulta una estructura estable que permita una división de funciones y tareas regulada y permanente. De ahí que uno de los objetivos prioritarios pase por el fortalecimiento institucional a todos los niveles a partir de la elaboración de un Plan Estratégico que sirva como guía de actuación y mejora de sus recursos ${ }^{27}$. Esta preocupación se canaliza a través de otros mecanismos como la convocatoria para la realización de un Taller de Fortalecimiento de la Coordinadora Nacional de Mujeres Indígenas de Panamá (agosto 2010) en el que se reunieron las representantes de los siete pueblos indígenas de Panamá, procedentes de sus respectivas Comarcas y Comunidades.

«Aunque la CONAMUIP sigue existiendo por las mujeres que están aquí. Son compañeras que están por un compromiso con su pueblo, con sus comunidades, con las mujeres, son la mayoría, son voluntarias, entonces yo siento que está lo débil porque hay compañeras que están pero por necesidad tiene que tener un trabajo y se van... pero aunque trabajan fuera, ellas llegan cuando están sus días libres, viene a aportar. Yo siento que el día que nos desaparezcamos sería por los recursos económicos porque no hay un apoyo» (Sonia Henríquez).

Un relato como el efectuado hasta aquí supone un intento de contribuir a la visibilización de algunos retales de la vida de una organización de mujeres indígenas. Pero no es posible concluir sin preguntarnos qué ha supuesto a lo largo de estos años su presencia pública en muy distintos foros y espacios políticos. En este punto retomo la reflexión de Dolores Juliano cuando plantea que en muchas ocasiones las estrategias de visibilización concluyen en situaciones de empoderamiento. La respuesta de las propias mujeres de la Coordinadora es que la organización ha sido una escuela de formación. Expresado en sus términos, una escuela para su propia identidad como personas que las ha fortalecido como mujeres, independiente de su condición de mujeres indígenas. La respuesta es la que formula Sonia Henríquez:

«Entonces aunque haya fuerza aunque haya capacidad cuando nos reunimos con otras mujeres que no son indígenas... a veces hay choques y eso también depende de nosotras de si estamos muy empoderadas y ahí viene lo que es el empoderamiento de la mujer, entonces nosotras sentimos que el estar en una Coordinadora Nacional de la Mujer, que estar en ese espacio, es ser partícipe de ese proceso, sentimos que tenemos ese empoderamiento... ahora es llevarlo, las mujeres en las comunidades es

\footnotetext{
vestidos tradicionales en diferentes espacios sociales y particularmente en sus respectivos ámbitos de trabajo.

$27 \mathrm{Su}$ objetivo principal es fortalecer el protagonismo político y social de la CONAMUIP para impulsar propuestas de desarrollo y democracias que garanticen la plena vigencia de los Derechos Humanos de las Mujeres Indígenas y la niñez en Panamá. Los enfoques que sustentan el Plan son: la transversalidad de la perspectiva de género, el enfoque de derechos humanos, el enfoque del ciclo de vida, el enfoque de interculturalidad, el enfoque de la transmisión intergeneracional de la violencia doméstica y el enfoque de desarrollo humano sostenible.
} 
lo que tiene que sentirse. Ese empoderamiento no es un mal, es un bien porque ante la faz nacional como indígenas que somos... también tenemos derechos como mujeres indígenas, como pueblos indígenas. Eso parte de un empoderamiento, de poder exigir nuestros derechos reivindicativos, también cuando algo no nos gusta, cuando sentimos que estamos siendo discriminadas... ahí está la voz de la mujer indígena. Yo siento que también ha servido para eso la CONAMUIP».

\section{Conclusiones}

La necesidad de establecer distintos planos en el conocimiento y comprensión de la Coordinadora de Mujeres Indígenas de Panamá se corresponde con la complejidad de sus propios procesos. La Coordinadora se crea en un contexto de emergencia paralela de diferentes Movimientos. Por un lado, su constitución tiene como marco la etapa de nuevo impulso del Movimiento de Mujeres de Panamá, concretado en la alianza Foro, Mujer y Desarrollo, cuyo objetivo será incidir en las políticas públicas en el proceso de construcción de nuevos modelos de democracia, con la participación plena de las mujeres que se concreta en la aparición de instituciones como el Consejo Nacional de la Mujer (CONAMU) y la Dirección Nacional de la Mujer (DINAMU), concebidas como mecanismos institucionales que promuevan políticas de igualdad entre hombres y mujeres. Estos espacios van a propiciar el encuentro entre las propias mujeres indígenas y será un contexto que va a facilitar el acuerdo que tendrá como consecuencia el nacimiento de la Coordinadora. Por otro lado, es el momento de emergencia de los pueblos indígenas lo que va a favorecer la creación del Enlace Continental de Mujeres Indígenas de las Américas (ECMIA) como un espacio pionero de encuentro de las mujeres indígenas a nivel continental. Esa confluencia de intereses diversos cristaliza y se canaliza en Panamá a través de la constitución de la CONAMUIP que, por otro lado es, fundamentalmente, la respuesta a sus demandas como mujeres, como indígenas (kuna, emberá, wounaan, ngobe, buglé, nasoy, bri-bri) y como panameñas. Su camino de consolidación de casi dos décadas de funcionamiento transcurre paralelo y al mismo tiempo entrecruzado con ambos Movimientos, generando un espacio de interlocución permanente que está también en la base de su discurso actual. Un discurso que destaca sobre todo su carácter de organización interétnica basada en las diferentes pertenencias étnicas y sus propias identidades culturales con participación plena de todos los pueblos indígenas de Panamá. Todo ello concebido como la base en la que se apoya el trabajo realizado con y desde las mujeres indígenas en todos los lugares en los que estas se encuentren (comunidades, comarcas, barriadas) favoreciendo procesos de empoderamiento individual y colectivo. Un discurso que incorpora, también, aquellas propuestas externas que son herramientas útiles para sus propios intereses lo que hace que en el momento actual trabajen con el trasfondo del enfoque transversal de género desde sus propias posiciones y situaciones socioculturales y económicas y desde sus específicos intereses prácticos y estratégicos de género. Ellas han conseguido consolidar un espacio que, sin embargo, no está exento de problemas y peligros en 
su propio funcionamiento. De ahí que una de las preocupaciones fundamentales en el momento actual sea poner en marcha mecanismos de fortalecimiento institucional como una línea estratégica que apuntale algunas de las posibles quiebras y propicie la continuidad de la Coordinadora. Así se ha constituido una organización de carácter integral que asume todos aquellos temas que afecten a los pueblos indígenas y que quiere hacer oír su voz y su opinión (ese discurso generado desde sus propias posiciones y situaciones) como mujeres indígenas y cuya incesante actividad y cuya presencia pública las convierte, cada vez más, en referentes de los pueblos indígenas de Panamá.

\section{Referencias bibliográficas}

Arce, Mariela

2001 «Las mujeres panameñas entre luces y sombras: avances, límites y desafíos de los procesos de incidencia política para institucionalizar el género en las políticas de desarrollo», en Políticas públicas para la equidad de género: una propuesta del movimiento de mujeres en Panamá, Y. Alaníz Chiari et al., pp. 43-74. Panamá: Instituto de la Mujer - Universidad de Panamá.

HERnÁndez, Rosalva Aida

2008 «Feminismos poscoloniales: reflexiones desde el sur de Río Bravo», en Descolonizando el feminismo. Teoría y práctica desde los márgenes, Liliana Suárez Navas y Rosalva Aida Hernández, eds., pp. 75-113. Madrid: Cátedra.

IV InForme NACIONAL «Clara GonZÁLEZ»

2008 Situación de la Mujer en Panamá. Panamá: Dirección Nacional de la Mujer, Ministerio de Desarrollo Social.

JuLIANO, Dolores

1992 El juego de las astucias. Mujer y construcción de modelos sociales alternativos. Madrid: Horas y HORAS.

Moore, Henrietta L.

1991 Antropología y feminismo. Madrid: Cátedra.

PizARro, Alibel

2001 Identidad y liderazgo. Panamá: Centro de Estudios y Acción Social Panameña. 